\title{
DARBOUX POLYNOMIALS, BALANCES AND PAINLEVÉ PROPERTY
}

\author{
JAUME LLIBRE ${ }^{1}$ AND CLÀUDIA VALLS ${ }^{2}$
}

\begin{abstract}
For a given polynomial differential system we provide different necessary conditions for the existence of Darboux polynomials using the balances of the system and the Painlevé property.
\end{abstract}

\section{IntRoduCtion AND STATEMENT OF THE MAIN RESUlts}

The Painlevé property appears studying the general solutions of the differential equations viewed as functions of complex time. More precisely, when the solutions are single-valued on its maximum domain of analytic continuation, we say that the system has the Painlevé property. In other words a differential system has the Painlevé property if its general solution has no movable critical singularities, see for more details [3]. This property imposes strong conditions, that despite the fact that it has not been proved, one believes that in this case the system is integrable. However there is no a precise algorithm to decide whether a system has the Painlevé property, and only necessary conditions can be obtained, called the Painlevé test. Most of the systems do not satisfy the Painlevé test but there is a lot of information concerning the global behavior of the system that we can obtain from the local analysis around the singularities in complex time and the lack of meromorphicity can be used to prove the nonintegrability of the system with meromorphic first integrals.

For more than half a century after its development, the Painlevé theory for differential equations was considered an interesting, if not old fashioned important part of the theory of special functions and little attention was paid to it until the early 1980's when it was discovered its relation with soliton theories. Since then, there has been a huge amount of works relating the Painlevé property with different branches of differential systems such as the integrabiity of PDE, the rational and polynomial integrability of ODE's ,... However very few is known about its relation with the Darboux theory of integrability for polynomial differential systems. The main aim of this pape is to focus on the connections between the existence of Darboux polynomials, the Painlevé property and the Kovalevskaya exponents (introduced

2010 Mathematics Subject Classification. Primary 34A05. Secondary 34C05, 37C10.

Key words and phrases. Painlevé property, Darboux polynomial, Kovalevskaya exponents. 
by Sophia Kowalevskaya to compute the Laurent series solutions of the rigid body motion).

In order to state the main results of the paper we consider a polynomial differential system of the form

$$
\frac{d x}{d t}=\dot{x}=P(x) \quad \text { with } x=\left(x_{1}, \ldots, x_{n}\right) \in \mathbb{C}^{n},
$$

and $P(x)=\left(P_{1}(x), \ldots, P_{n}(x)\right)$ and $P_{i} \in \mathbb{C}\left[x_{1}, \ldots, x_{n}\right]$ for $i=1, \ldots, n$. As usual $\mathbb{C}$ denotes the set of complex numbers, and $\mathbb{C}\left[x_{1}, \ldots, x_{n}\right]$ denotes the polynomial ring over $\mathbb{C}$ in the variables $x_{1}, \ldots, x_{n}$. Here $t$ can be real or complex. The maximum of the degrees of the polynomials $P_{i}$ for $i=1, \ldots, n$ is called the degree of the polynomial differential system (1).

Assume that there exists a solution of the form

$$
x=\alpha t^{p}, \quad \text { i.e. } \quad\left(x_{1}, \ldots, x_{n}\right)=\left(\alpha_{1} t^{p_{1}}, \ldots, \alpha_{n} t^{p_{n}}\right)
$$

where $p=\left(p_{1}, \ldots, p_{n}\right), \alpha=\left(\alpha_{1}, \ldots, \alpha_{n}\right) \neq(0, \ldots, 0), \alpha_{i} \in \mathbb{C}$ and $p_{i} \in \mathbb{R}$ are given by one of the non-vanishing solutions of the algebraic equation

$$
p_{i} \alpha_{i}=P_{i}(\alpha), \quad i=1, \ldots, n .
$$

For a given $p$ there may exist different sets of values of $\alpha$, called balances. The Kowalevskaya matrix associated to a balance $\alpha$ is

$$
M(\alpha)=D P(\alpha)-\operatorname{diag}\left(p_{1}, \ldots, p_{n}\right),
$$

where as usual $D P(\alpha)$ denotes the Jacobian matrix of $P$ evaluated at $\alpha$ and $\operatorname{diag}\left(p_{1}, \ldots, p_{n}\right)$ denotes the matrix whose diagonal is equal to $\left(p_{1}, \ldots, p_{n}\right)$ and zeroes in the rest. The eigenvalues of the matrix $M$ are called the $\mathrm{Ko}$ valevskaya exponents of the balance $\alpha$ and are denoted by $\rho=\left(\rho_{1}, \ldots, \rho_{n}\right)$. It can be shown that there always exists a Kowalevskaya exponent equal to -1 related to the arbitrariness of the origin of the parameterizations of the solution by the time. The eigenvector associated to the eigenvalue $\rho_{1}=-1$ is $p \alpha=\left(p_{1} \alpha_{1}, \ldots, p_{n} \alpha_{n}\right)$. For more details see [3] or [2].

In section 2 we recall how to compute the solutions of the form (2) when the polynomial differential system is quasi-homogeneous.

A non-constant polynomial $F\left(x_{1}, \ldots, x_{n}\right)$ is a Darboux polynomial of system (1) if it satisfies

$$
\sum_{i=1}^{n} P_{i} \frac{\partial F}{\partial x_{i}}=P \cdot \nabla F=K F
$$

where $K \in \mathbb{C}\left[x_{1}, \ldots, x_{n}\right]$ is called the cofactor and has degree at most $m-1$, if $m$ is the degree of the polynomial differential system (1). As usual $\nabla F$ denotes the gradient of the function $F$. We recall that $F$ is a Darboux polynomial if and only if $F(x)=0$ is an invariant hypersurface of system (1), i.e. if a solution of system (1) has a point on the hypersurface $F(x)=0$, then the whole solution is contained in this hypersurface. 
A polynomial $F$ is said to be weight-homogeneous if there exists $d \in \mathbb{Q}$ and $s=\left(s_{1}, \ldots, s_{n}\right) \in \mathbb{Q}^{n}$ such that for an arbitrary positive real $a$ we have

$$
F\left(a^{s_{1}} x_{1}, \ldots, a^{s_{n}} x_{n}\right)=a^{d} F\left(x_{1}, \ldots, x_{n}\right) .
$$

Here $d$ is called the weight degree of the polynomial $F$ and $s$ is the weight exponent of $F$.

The first result of this paper is the following.

Theorem 1. Assume that the polynomial differential system (1) admits the particular solution $x=\alpha t^{p}$ given in (2). If $F(x)$ is a weight-homogeneous Darboux polynomial of weight degree $d$ of system $(1)$, then $\nabla F(\alpha) \neq 0$ and its cofactor $K$ cannot be constant.

The proof of Theorem 1 is given in section 4 .

Theorem 1 is closely related with Theorem 5.4 of [3] which is due to Yoshida (see [7, 8]) and states: Under the assumptions of Theorem 1 if $I(x)$ is a weight-homogeneous first integral of weight degree $d$ of system (1) satisfying that $\nabla I(\alpha) \neq 0$, then $d$ is a Kowalevskaya exponent of the matrix $M(\alpha)$ given in (4).

For the second result we need some more definitions and notation. We can write the polynomial $P_{i}(x)$ for $i=1, \ldots, n$ in the form

$$
P_{i}(x)=\sum_{j=0}^{m_{i}} P_{i}^{(j)}(x)
$$

where $P_{i}^{(j)}(x)$ is a weight-homogeneous polynomial of weight exponent $p \in$ $\mathbb{Q}^{n}$ with weight degree $p_{i}+q^{(j)}-1$, i.e.

$$
P_{i}^{(j)}\left(t^{p} x\right)=t^{p_{i}+q^{(j)}-1} P_{i}^{(j)}(x)
$$

for $i=1, \ldots, n$ and $j=0,1, \ldots, m_{i}$, with $q^{(j)} \in \mathbb{Q}$ and $q^{(0)}=0<q^{(1)}<$ $\cdots<q^{(m)}$, where $m$ is the maximum of the $\left\{m_{1}, \ldots, m_{n}\right\}$.

Assume that $\dot{x}=P^{(0)}(x)$ has the solution $x=\alpha t^{p}$ where $t=t-t_{*}$ for some complex $t_{*}$, and $\alpha \in \mathbb{C}^{n}$ with $|\alpha|=\left|\alpha_{1}\right|+\cdots+\left|\alpha_{n}\right| \neq 0$. Then we say that the polynomial differential system (1) admits a dominant balance $\{\alpha, p\}$.

We note that any balance of system (1) is a dominant balance taking $P_{i}^{(0)}=P_{i}$. An example of a polynomial differential system with a dominant balance can be found in section 3 .

Now we study the relation between the Kovalevskaya exponents and Darboux polynomials.

Theorem 2. Assume that the polynomial differential system (1) admits a dominant balance $\{\alpha, p\}$ such that the Kovalevskaya matrix

$$
M(\alpha)=D P^{(0)}(\alpha)-\operatorname{diag}\left(p_{1}, \ldots, p_{n}\right),
$$


diagonalizes. Then the following statements hold.

(a) If the eigenvalues $\left(\rho_{1}, \ldots, \rho_{n}\right)$ (the Kovalevskaya exponents ) of $M(\alpha)$ are $\mathbb{Z}$-independent and $F(x)$ is a Darboux polynomial of system (1), then $F(x)$ must have a cofactor $K$ such that $K^{(0)}(\alpha)$ is not a rational number.

(b) If the Kovalevskaya exponents $\left(\rho_{1}, \ldots, \rho_{n}\right)$ of $M$ are $\mathbb{N}$-independent and $F(x)$ is a Darboux polynomial of system (1), then $F(x)$ must have a cofactor $K$ such that $K^{(0)}(\alpha) \notin\{0,1,2,3, \ldots\}$.

Moreover we explore some connections between Painlevé property and Darboux polynomials. The first result connecting the Painlevé property with the Darboux polynomials is the following.

Theorem 3. Assume that the polynomial differential system (1) satisfies the Painlevé property. Then if the system has a Darboux polynomial its cofactor $K$ must satisfy $K(\alpha) \in \mathbb{Z}$ for all balances $\alpha$ of the system.

We also consider a kind of converse result of Theorem 3 .

Theorem 4. Assume that the polynomial differential system (1) admits a dominant balance $\{\alpha, p\}$ and it has a Darboux polynomial with cofactor $K$ such that $K^{(0)}(\alpha) \notin \mathbb{Z}$. Then system (1) cannot satisfy the Painlevé property.

The proofs of Theorems 2, 3 and 4 are given in section 5 .

See section 6 for some examples of systems satisfying the conditions of Theorems 1, 2, 3 and 4 and the conclusion section for some comments on our results.

\section{QUASI-HOMOGENEOUS POLYNOMIALS DIFFERENTIAL SYSTEMS}

The polynomial differential system (1) is quasi-homogeneous if there exists $s=\left(s_{1}, \ldots, s_{n}\right) \in \mathbb{N}^{n}$ and $r \in \mathbb{N}$ such that for an arbitrary positive real $a$ we have

$$
P_{i}\left(a^{s_{1}} x_{1}, \ldots, a^{s_{n}} x_{n}\right)=a^{s_{i}-1+r} P_{i}\left(x_{1}, \ldots, x_{n}\right)
$$

for $i=1, \ldots, n$. We call $s=\left(s_{1}, \ldots, s_{n}\right)$ the weight exponent of system (1) and $r$ the weight degree with respect to the weight exponent $s$. In particular the case that $s=(1, \ldots, 1)$ we say that system (1) is a homogeneous polynomial differential system of degree $r$.

If a polynomial differential system (1) is quasi-homogeneous with weight exponent $s$ and weight degree $r>1$ then the system is invariant under the change of variables

$$
x_{i} \rightarrow a^{-p_{i}}, \quad t \rightarrow a^{-1} t
$$


where

$$
p_{i}=s_{i} /(1-r) .
$$

This fact implies that in this case there exists solutions of the form (2) with $p_{i}=s_{i} /(1-r)$ for $i=1, \ldots, n$ and the coefficients $\alpha_{i}$ satisfying (3). The integrability of quasi-homogeneous polynomial differential systems have been investigated by several authors, see for instance $[2,3,5,6,8,9,10]$.

\section{Dominant Balances}

Consider the polynomial differential system (1). We select a suitable weight change of variables denoted by $X=\left(X_{1}, \ldots, X_{n}\right)=a^{s} x=\left(a^{s_{1}} x_{1}, \ldots\right.$, $a^{s_{n}} x_{n}$ ) where $s_{i} \in \mathbb{N}$ for $i=0, \ldots, n$ such that (1) becomes

$$
\dot{X}_{i}=a^{-s_{0}} \sum_{j=0}^{r} a^{j} P_{i, m-j}(X), \quad \text { for } i=1, \ldots, n,
$$

where $r \leq m=\operatorname{degree}(P)$ and $P_{i, m-j}$ is a weight-homogeneous polynomial. In particular $P_{i, m}(X)=P_{i}^{(0)}(x)$ and $P_{i, m-j}(X)=P_{i}^{(j)}(x)$ for $j=1, \ldots, r$. In this case $p=\left(s_{1}, \ldots, s_{n}\right)$.

Consider the Lorenz system

$$
\begin{aligned}
& \dot{x}_{1}=s\left(x_{2}-x_{1}\right), \\
& \dot{x}_{2}=r x_{1}-x_{2}-x_{1} x_{3}, \\
& \dot{x}_{3}=-b x_{3}+x_{1} x_{2},
\end{aligned}
$$

where $s, r, b$ are real parameters and $s \neq 0$. We make the change of variables

$$
x_{1}=a^{-1} X_{1}, \quad x_{2}=a^{-2} X_{2}, \quad x_{3}=a^{-2} X_{3}
$$

system (9) becomes

$$
\begin{aligned}
& \dot{X}_{1}=a^{-1} s\left(X_{2}-a X_{1}\right), \\
& \dot{X}_{2}=a^{-1}\left(-X_{1} X_{3}-a X_{2}+r a^{2} X_{1}\right), \\
& \dot{X}_{3}=a^{-1}\left(X_{1} X_{2}-b a X_{3}\right) .
\end{aligned}
$$

Equation (10) implies that

$$
P^{(0)}=\left(s x_{2},-x_{1} x_{3}, x_{1} x_{2}\right), \quad P^{(1)}=\left(-s x_{1},-x_{2},-b x_{3}\right), \quad P^{(2)}=\left(0, r x_{1}, 0\right) .
$$

Two dominant balances for system (9) are $\{\alpha, p\}$ with $\alpha=(-2 i, 2 i / s,-2 / s)$ and $p=(-1,-2,-2)$; and $\{\alpha, p\}$ with $\alpha=(2 i,-2 i / s,-2 / s)$ and $p=$ $(-1,-2,-2)$. Note that here $q^{(1)}=1$ and $q^{(2)}=2$. 


\section{PROOF OF THEOREM 1}

We first introduce two auxiliary results.

Lemma 5. Let $F(x)$ be a Darboux polynomial with cofactor $K$ of system (1) and $\hat{x}(t)=\left(\hat{x}_{1}(t), \ldots, \hat{x}_{n}(t)\right)$ a particular solution of system (1). If $K=$ $k_{0} \in \mathbb{C}^{n}$, then $\bar{u}(t)=e^{-k_{0} t} \nabla F(\hat{x}(t))$ is a solution of the adjoint equation, that is,

$$
\dot{\bar{u}}=-D P(\hat{x}(t) \bar{u}
$$

Proof. Let $H F$ be the Hessian of the matrix of the polynomial $F$. To show that $\bar{u}$ is a solution of the adjoint equation, we compute its time derivative. Note that

$$
\begin{aligned}
\dot{\bar{u}}= & \frac{d}{d t} \bar{u}=\frac{d}{d t}\left(e^{-k_{0} t} \nabla F(\hat{x}(t))\right. \\
= & e^{-k_{0} t} \frac{d}{d t}\left(\nabla F(\hat{x}(t))-k_{0} e^{-k_{0} t} \nabla F(\hat{x}(t))\right. \\
= & e^{-k_{0} t} H F(\hat{x}(t)) \cdot P(\hat{x}(t))-k_{0} e^{-k_{0} t} \nabla F(\hat{x}(t)) \\
= & e^{-k_{0} t} \nabla(P(\hat{x}(t)) \cdot \nabla F(\hat{x}(t)))-e^{-k_{0} t} D P(\hat{x}(t)) \nabla F(\hat{x}(t)) \\
& -k_{0} e^{-k_{0} t} \nabla F(\hat{x}(t)) \\
= & -D P(\hat{x}(t))\left(e^{-k_{0} t} \nabla F(\hat{x}(t))\right) \\
= & -D P(\hat{x}(t)) \bar{u} .
\end{aligned}
$$

This completes the proof of the lemma.

The following lemma will be the central tool in the nonintegrability results. Its proof uses Lemma 5.

Lemma 6. Let $F(x)$ be a Darboux polynomial with cofactor $K=k_{0} \in \mathbb{C}^{n}$ of system (1). For a solution $\bar{x}(t)$ of the polynomial differential system (1), let $u(t)$ be the solution of the variational equation $\dot{u}=D P(\hat{x}(t)) u$. Then the function $I=e^{-k_{0} t} \nabla F(\hat{x}(t)) \cdot u$ is a Darboux invariant of this variational equation.

Proof. We compute the time derivative of $I$ and we show that it is zero. Indeed, by Lemma 5

$$
\begin{aligned}
\frac{d I}{d t} & =\frac{d}{d t}\left(e^{-k_{0} t} \nabla F(\hat{x}(t)) \cdot u\right) \\
& =\frac{d}{d t}\left(e^{-k_{0} t} \nabla F(\hat{x}(t))\right) \cdot u+\left(e^{-k_{0} t} \nabla F(\hat{x}(t))\right) \cdot \frac{d u}{d t} \\
& =-e^{-k_{0} t}(D P(\hat{x}(t)) \nabla F(\hat{x}(t))) \cdot u+\left(e^{-k_{0} t}(D P(\hat{x}(t)) \nabla F(\hat{x}(t))) \cdot u\right. \\
& =0 .
\end{aligned}
$$

This completes the proof of the lemma. 
Now we continue with the proof of the theorem. We proceed by contradiction. Consider system (1) which admits the particular solution $x=\alpha t^{p}$. Assume that it has a weight-homogeneous Darboux polynomial $F(x)$ such that $\nabla F(\alpha) \not \equiv 0$ and with cofactor $K=k_{0}$. We can apply Lemma 6 . The existence of $F(x)$ implies the existence of a first integral $I=e^{-k_{0} t} \nabla F\left(\alpha t^{p}\right) u \not \equiv 0$ for the variational equation

$$
\dot{u}=D P\left(\alpha t^{p}\right) u .
$$

The general solution of the variational equation is of the form $u=\sum_{i=1}^{k} \beta^{(i)} t^{\rho_{i}}$ where $\beta^{(i)}$ is a polynomial in $\log t$ and can be expressed in terms of the generalized eigenvectors of the Kowalevskaya matrix $K=D f(\alpha)-\operatorname{diag}\left(p_{1}, \ldots, p_{n}\right)$ and $\rho_{i}$ are the Kowalevskaya exponents, for more details see section 3.8.2 of [3]. This general solution contains $n$ arbitrary parameters. We can therefore evaluate $I$ on this solution to obtain

$$
I=\sum_{i=1}^{k} e^{-k_{0} t} t^{-d+\rho_{i}} \nabla F(\alpha) \beta^{(i)} .
$$

If $K$ is semi-simple (i.e., it can be diagonalized), then $k=n$ and the eigenvectors $\beta^{(i)}$ form a set of $n$ linearly independent vectors which at least one of them, say $i=j$ is such that $\nabla F(\alpha) \beta^{(j)} \neq 0$, otherwise $I$ would be zero. Since $I$ is constant in time, we get a contradiction.

If $K$ is not semi-simple, then there is a complete set of generalized eigenvectors $\gamma^{(1)}, \ldots, \gamma^{(n)}$ for $K$ for which at least one of them satisfies $\nabla F(\alpha) \gamma^{(i)} \neq$ 0 and the contradiction follows. This completes the proof of the theorem.

\section{Proof of the Remaining Results}

In this section we provide the proof of Theorems 2,3 and 4 . To prove Theorem 2 we will use the following theorem which is Theorem 5.7 in [3]. We continue to decompose any polynomial as in (6).

Theorem 7. Assume that the polynomial differential system (1) has a Darboux polynomial $F$ with cofactor $K$ and a dominant balance $\{\alpha, p\}$ with Kovalevskaya exponents $\rho=(-1, \tilde{\rho})$ with $\tilde{\rho} \in \mathbb{C}^{n-1}$. Then there exists a vector $m=\left(m_{2}, \ldots, m_{n}\right)$ of positive integers such that

$$
\tilde{\rho} \cdot m=d+K^{(0)}(\alpha)
$$

where $d=\operatorname{deg}\left(F^{(0)}\right)$ and $|m| \leq d$.

Proof of Theorem 2. The proof of both statements will be done by contradiction.

To prove statement (a) we assume that there is a Darboux polynomial $F(x)$ such that $K^{(0)}(\alpha)$ is a rational number. Then it follows from Theorem 7 that there exist integers $i_{2}, \ldots, i_{m}$ such that $i_{2} \rho_{2}+\ldots+i_{n} \rho_{n}=\tilde{d}$ where 
$\tilde{d} \in \mathbb{Q}$. This relation can be written as $j_{1} \rho_{1}+j_{2} \rho_{2}+\ldots+j_{n} \rho_{n}=0$ for some integers $j_{1}, \ldots, j_{n}$. However this is impossible because $\rho_{1}, \ldots, \rho_{n}$ are $\mathbb{Z}$-independent.

To prove statement (b) we assume that there is a Darboux polynomial $F(x)$ such that $K^{(0)}(\alpha)$ is a non-negative integer. Then it follows from Theorem 7 that there exist integers $i_{2}, \ldots, i_{m}$ such that $i_{2} \rho_{2}+\ldots+i_{n} \rho_{n}=\tilde{d}$ where $\tilde{d} \in \mathbb{Z}$. This relation can be written as $\tilde{d} \rho_{1}+i_{2} \rho_{2}+\ldots+i_{n} \rho_{n}=0$ for some naturals $i_{1}, \ldots, i_{n}$. However this is impossible because $\rho_{1}, \ldots, \rho_{n}$ are $\mathbb{N}$-independent.

To prove Theorem 3 we will use again Theorem 7 and the following result proved in [3].

Proposition 8. Consider a system $\dot{x}=f(x)$ where $f(x)$ is analytic and assume that it satisfies the Painlevé property. Then for all possible balances $\{\alpha, p\}$ the Kovalevskaya matrix $K$ diagonalizes and the Kovalevskaya exponents $\left(\rho_{1}, \ldots, \rho_{n}\right)$ are integers.

Proof of Theorem 3. It follows from Proposition 8 that for all possible balances $\{\alpha, p\}$ the Kovalevskaya matrix $K$ diagonalizes and the Kovalevskaya exponents $\rho=\left(-1, \rho_{2}, \ldots, \rho_{n}\right)=(-1, \tilde{\rho})$ are integers. Now we apply Theorem 7 and from the notation and definitions introduced there we conclude that

$$
K(\alpha)=\tilde{\rho} \cdot m-d \in \mathbb{Z},
$$

and the proof is completed.

Proof of Theorem 4. It follows from Theorem 7 that $\tilde{\rho} \cdot m \notin \mathbb{Z}$ (we have used the notations and definitions introduced there). Therefore there must exists at least one Kovalevskaya exponent which is not an integer number, and so it follows from Proposition 8 that system (1) cannot satisfy the Painlevé property.

\section{EXAMPLES}

6.1. Example for Theorem 1. Consider the system

$$
\begin{aligned}
& \dot{x}_{1}=-x_{3}, \\
& \dot{x}_{2}=-x_{4}, \\
& \dot{x}_{3}=3 i\left(x_{2}-i x_{1}\right)\left(x_{2}+i x_{1}\right)^{2}-i\left(x_{2}+i x_{1}\right)^{3}, \\
& \dot{x}_{4}=3\left(x_{2}-i x_{1}\right)\left(x_{2}+i x_{1}\right)^{2}+i\left(x_{2}+i x_{1}\right)^{3} .
\end{aligned}
$$

Note that it is a quasi-homogeneous polynomial differential system with weight exponent $s=(1,1,2,2)$ and weight degree $r=2$, see section 2 . 
System (14) has the Darboux polynomials

$$
F_{1}(x)=x_{3}-i x_{4}-\left(x_{2}+i x_{1}\right)^{2}, \quad F_{2}=x_{3}-i x_{4}+\left(x_{2}+i x_{1}\right)^{2}
$$

which are both weight-homogeneous with weight degree 2 . The cofactor $K_{1}$ of $F_{1}$ is $-2\left(x_{1}-i x_{2}\right)$ and the cofactor $K_{2}$ of $F_{2}$ is $2\left(x_{1}-i x_{2}\right)$. None of these cofactors are constant and $\nabla F_{j}(\alpha) \neq 0$ for $j=1,2$, as stated in Theorem 1. Note that it is not necessary to compute the $\alpha$ corresponding to $p=-s$ because $\nabla F_{j}=(*, *, 1,-i)$.

6.2. Example for Theorem 2. Now consider the Lorenz polynomial differential system (9) of section 3 . We recall that a dominant balance for system (9) is $\{\alpha, p\}$ with $\alpha=(-2 i, 2 i / s,-2 / s)$ and $p=(-1,-2,-2)$. Now we compute the matrix $M(\alpha)$ of (8) and we get

$$
M(\alpha)=\left(\begin{array}{ccc}
1 & s & 0 \\
2 / s & 2 & 2 i \\
2 i / s & -2 i & 2
\end{array}\right),
$$

whose eigenvalues are $-1,2,4$. For the other dominant balance $\{\alpha, p\}$ with $\alpha=(2 i,-2 i / s,-2 / s)$ and $p=(-1,-2,-2)$ we have that

$$
M(\alpha)=\left(\begin{array}{ccc}
1 & s & 0 \\
2 / s & 2 & -2 i \\
-2 i / s & 2 i & 2
\end{array}\right),
$$

whose eigenvalues are again $-1,2,4$. Note that these Kowalevskaya exponents are $\mathbb{N}$-dependent.

On the other hand for a non-negative integer $n$ the Lorenz system with $s=-n / 2$ and $b=2 s$ has the Darboux polynomial $x_{1}^{2}-2 s x_{3}$ with cofactor $K=K^{(0)}(\alpha)=n$. This Lorenz system satisfies the contrapositive of statement (b) of Theorem 2.

6.3. Example for Theorem 3. Consider the polynomial differential system

$$
\dot{x}_{1}=x_{1}^{2}, \quad \dot{x}_{2}=x_{1}^{2} .
$$

The general solution of this system is

$$
x_{1}(t)=-\frac{1}{t+c_{1}}, \quad x_{2}(t)=c_{2}-\frac{1}{t+c_{1}},
$$

with $c_{1}$ and $c_{2}$ constant. Since this general solution is single-valued on its maximum domain of analytic continuation in $\mathbb{C}$, system (15) satisfies the Painlevé property.

System (15) is quasi-homogeneous with weight exponent $(1,1)$ and weight degree 2. So $p=(-1,-1)$ and the system has a solution of the form $\alpha t^{p}$ with $\alpha=(-1,-1)$, see for more details section 3. Moreover this system has the Darboux polynomial $F=x_{1}$ with cofactor $K=x_{1}$. Therefore $K(\alpha)=-1 \in \mathbb{Z}$. So this differential system satisfies Theorem 3 . 
6.4. Example for Theorem 4. The Lorenz system with the parameters $s=1 / 3$ and $b=0$ does not satisfy the Painlevé property, see for details [4]. This system has the Darboux polynomial $x_{1}^{4}-4 x_{1}^{2} x_{3} / 3-4 x_{2}^{2} / 9-8 x_{1} x_{2} / 9+$ $4 r x_{1}^{2} / 3$ with cofactor $K=-4 / 3 \notin \mathbb{Z}$. So it also satisfies Theorem 4 .

Furthermore the Lorenz system with the parameters $s=1 / 2, b=1$ and $r=0$ satisfies the Painlevé property, see again [4]. This system has the Darboux polynomials $x_{1}^{2}-2 s x_{3}$ and $x_{2}^{2}+x_{3}^{2}$ with cofactors $-2 s=-1 \in \mathbb{Z}$ and $-2 \in \mathbb{Z}$, respectively. So this example satisfies the contrapositive statement of Theorem 4.

\section{Conclusions}

Theorem 1 provides two necessary conditions for the existence of a Darboux polynomial for the polynomial differential system (1), one of them related with the balances of the system.

Theorem 2 provides two necessary conditions for the existence of a Darboux polynomial of system (1), but now one of these conditions is related with the balances of the system $\dot{x}=P^{(0)}(x)$ instead of the balances of system (1).

Theorems 3 and 4 provide necessary conditions for the existence of a Darboux polynomial of system (1) using the Painlevé property.

Theorem 7 due to Goriely provides different necessary conditions for the existence of a Darboux polynomial of system (1) distinct to the ones given in Theorems 1-4.

\section{ACKNOWLEDGEMENTS}

The first author is partially supported by a FEDER-MINECO grant MTM2016-77278-P, a MINECO grant MTM2013-40998-P, and an AGAUR grant number 2014SGR-568. The second author is partially supported by FCT/Portugal through UID/MAT/04459/2013.

\section{REFERENCES}

[1] F. Dumortier, J. Llibre And J.C. Artés, Qualitative Theory of Planar Differential Systems, Springer Verlag, New York, (2006).

[2] S.D. FurTA, On non-integrability of general systems of differential equations, Z. Angew. Math. Phys. 47 (1996), 112-131.

[3] A. Goriely, Integrability and nonintegrability of dynamical systems, Advanced Series in Nonlinear Dynamics, 19. World Scientific Publishing Co., 2001.

[4] N.A. Kudryashov, Analytical solutions of the Lorenz system, Regular and Chaotic Dynamics 20 No. 2 (2015), 123-133.

[5] J. Llibre And X. Zhang, Polynomial first integrfals for quasi-homogeneous polynomial differential systems, Nonlinearity 15 (2002), 1269-1280. 
[6] A. Tsygvintsev, On the existence of polynomial first integrals of quadratic homogeneous systems of ordinary differential equations, J. Phys. A: Math. Gen. 34 (2001), $2185-2193$.

[7] H. Yoshida, Necessary conditions for existence of algebraic first integrals I, Celestial Mech. 31 (1983), 363-379, 381-399.

[8] H. Yoshida, Necessary conditions for existence of algebraic first integrals II, Celestial Mech. 31 (1983), 381-399, 381-399.

[9] H. Yoshida, A note on Kowalevski exponents and the non-existence of an additional analytic integral, Celestial Mech. 44 (1988), 313-316.

[10] H. YoshidA, A criterion for the non-existence of an additional analytic integral in Hamiltonian systems with $n$ degrees of freedom, Phys. Lett. A 141 (1989), 108-112.

${ }^{1}$ Departament de Matemàtiques, Universitat Autònoma de Barcelona, 08193 Bellaterra, Barcelona, Catalonia, Spain

E-mail address: jllibre@mat.uab.cat

2 Departamento de Matemática, Instituto Superior Técnico, Universidade Técnica de Lisboa, Av. Rovisco Pais 1049-001, Lisboa, Portugal

E-mail address: cvalls@math.ist.utl.pt 